\title{
Um framework para a adaptação de jogos sérios com Computação Afetiva e Computação de Personalidade
}

\author{
Renan Vinicius Aranha \\ Escola Politécnica \\ Universidade de São Paulo \\ São Paulo, São Paulo, Brasil \\ Email: renanvinicius@usp.br
}

\author{
Fátima L. S. Nunes \\ Escola de Artes, Ciências e Humanidades \\ Universidade de São Paulo \\ São Paulo, São Paulo, Brasil \\ Email: fatima.nunes@usp.br
}

\begin{abstract}
Resumo-Atividades de treinamento, ensino-aprendizagem ou de reabilitação motora podem envolver grandes desafios para os usuários. Com o intuito de tornar tais procedimentos mais atrativos e envolventes, pesquisadores têm investigado o desenvolvimento de jogos sérios, que cujas objetivos vão além do entretenimento, podendo envolver aquisição de conhecimento ou habilidades. Dentre as abordagens aplicáveis a esta finalidade, destacase a adaptação automática do jogo conforme as características do jogador. Neste contexto, este projeto propõe o desenvolvimento de um framework que possibilite o desenvolvimento de jogos sérios automaticamente adaptáveis, conforme o estado emocional e os traços de personalidade do usuário. Espera-se que a abordagem proposta não apenas auxilie o desenvolvimento de jogos desta natureza, como também possibilite a manutenção do nível de engajamento do jogador.
\end{abstract}

\section{INTRODUÇÃO}

Visando a tornar atividades e processos mais atrativos aos usuários, diversas iniciativas promovem o desenvolvimento de jogos sérios [1]. O termo "jogos sérios" (serious games, em Inglês) é utilizado para designar uma classe de jogos que visa a oferecer não apenas entretenimento mas, também, oportunizar experiências que possibilitem o desenvolvimento de habilidades [2].

Devido ao caráter motivacional, a manutenção do engajamento do usuário é um fator importante para a eficácia deste tipo de aplicação computacional. Neste contexto, uma abordagem que apresenta-se como promissora para propiciar uma melhor experiência de uso e, consequentemente, promover a manutenção do engajamento do usuário consiste na adaptação automática de jogos sérios conforme as características do usuário. O framework EasyAffecta, proposto por Aranha et al. [3], propicia a adaptação automática de jogos sérios para reabilitação motora conforme o estado emocional do usuário. A concepção do framework prevê benefícios a pacientes, fisioterapeutas e programadores. Além de reduzir o custo de implementação ao programador, o framework possibilita que o fisioterapeuta configure as adaptações a serem efetuadas para cada paciente.

No entanto, embora a abordagem tenha apresentado resultados positivos [4], a análise manual das características dos usuários é um processo complexo, que: i) por ter caráter subjetivo, pode apresentar vieses e ser impreciso para diferentes usuários; e ii) demanda esforço do fisioterapeuta, profissional da área de aplicação, podendo ser inviável quando há um grande número de usuários [5]. Adicionalmente, esta abordagem apresenta como limitação o escopo de jogos sérios voltados ao processo de reabilitação motora.

\section{TRABALHOS RELACIONADOS}

A literatura científica apresenta outros estudos que investigam estratégias para a manutenção do engajamento do usuário neste tipo de aplicação. Em [6], os autores analisaram a adaptação de um jogo sério a partir dos traços de personalidade do usuário. Tadayon et al. [5], por outro lado, analisaram a adaptação de um jogo sério para aprendizado motor considerando os estados emocionais dos pacientes. Iniciativas que envolvem o desenvolvimento de jogos sérios com foco no usuário são apresentadas em [7] e [8].

Do conhecimento dos autores deste projeto, não há trabalhos que investiguem o desenvolvimento de uma abordagem que possibilite a adaptação automática de um jogo considerando tanto os traços de personalidade quanto o estado emocional do usuário.

\section{DEFINIÇÃO DA PROPOSTA}

Para propiciar a adaptação automática de jogos sérios conforme o estado emocional e os traços de personalidade do jogador, esta pesquisa prevê o aprimoramento do framework Easy Affecta. Proposto em [4], o framework é inicialmente composto por:

- um módulo de Reconhecimento, responsável por obter dados do usuário e inferir o estado emocional do mesmo;

- um módulo denominado Controlador Emoção - Ação, no qual o profissional da área de aplicação pode criar grupos de usuários e configurar as adaptações que devem ser executadas para cada grupo, conforme variações no estado emocional dos usuários; 
- um módulo de Adaptação, inserido na aplicação final, que recebe as instruções de adaptação do módulo Controlador Emoção-Ação e as executa no jogo.

A principal contribuição desta pesquisa, que acarretará no aprimoramento do framework, consiste na definição e implementação de estratégias para: i) a análise dos traços de personalidade do usuário; ii) a definição dos elementos de jogabilidade adaptáveis; e iii) a definição das adaptações recomendadas para um determinado usuário, considerando seu estado emocional e seus traços de personalidade.

Em decorrência destas ações, minimiza-se a necessidade de um agente intermediário para a configuração dos perfis de usuário, papel então exercido pelo fisioterapeuta na primeira versão do EasyAffecta. Finalmente, é válido destacar que, embora a prova de conceitos considere a adaptação afetiva de jogos sério, objetiva-se que a abordagem e o arcabouço computacional sejam úteis para adaptar aplicações de qualquer natureza.

\section{RESULTADOS PRELIMINARES}

Dentre os resultados preliminares, pode-se citar a publicação dos seguintes artigos: i) uma revisão sistemática de literatura sobre os impactos dos traços de personalidade na experiência do usuário em sistemas de Realidade Virtual [9]; e ii) uma revisão sistemática de literatura descrevendo as técnicas utilizadas para a adaptação automática de software a partir do estado emocional do usuário [10].

\section{Considerações Finais}

A partir da condução desta pesquisa de doutorado, prevêse o provimento de contribuições para as áreas que envolvem aplicações interativas, como Realidade Virtual e Aumentada, Jogos Sérios, Interação Humano-Computador e Computação Afetiva. Para que tais contribuições sejam possíveis, os seguintes resultados são esperados: i) a elaboração de uma abordagem que possibilite o reconhecimento da personalidade do usuário; ii) o desenvolvimento de um framework que possibilite a adaptação automática de jogos sérios considerando os traços de personalidade e o estado emocional dos usuários; iii) investigações sobre estratégias que possibilitem o relacionamento entre características do usuário e os elementos adaptáveis em software.

Complementarmente, a pesquisa descrita tem potenciais contribuições sociais, tecnológicas e econômicas. No aspecto social, a abordagem proposta pode contribuir com o aprimoramento de processos de treinamento, ensino-aprendizagem e reabilitação. Em relação aos aspectos tecnológicos, as contribuições deste trabalho envolvem a redução da complexidade de desenvolvimento de aplicações que envolvem a análise do estado emocional e dos traços de personalidade do usuário. Finalmente, os impactos econômicos decorrem da maior produtividade propiciada pelo arcabouço tecnológico disponibilizado.

\section{AGRADECIMENTOS}

O presente trabalho foi realizado com apoio da Coordenação de Aperfeiçoamento de Pessoal de Nível Superior - Brasil (CAPES) - Código de Financiamento 001. Os autores agradecem ao Conselho Nacional de Desenvolvimento Cientifico e Tecnológico (CNPq) (Processo 157535/2017-7) e à Fundação de Amparo à Pesquisa do Estado de São Paulo (FAPESP) (Processos 14/50889-7): Instituto Nacional de Ciência e Tecnologia em Medicina Assistida por Computação Científica (INCT-MACC).

\section{REFERÊNCIAS}

[1] S. Çiftci, "Trends of serious games research from 2007 to 2017: A bibliometric analysis," Journal of Education and Training Studies, vol. 6, no. 2, p. 18, jan 2018. [Online]. Available: https://doi.org/10.11114/jets.v6i2.2840

[2] R. Dörner, S. Göbel, W. Effelsberg, and J. Wiemeyer, Introduction. Cham: Springer International Publishing, 2016, pp. 1-34. [Online]. Available: https://doi.org/10.1007/ 978-3-319-40612-1_1

[3] R. V. Aranha, L. S. Silva, M. L. Chaim, and F. L. S. Nunes, "Using affective computing to automatically adapt serious games for rehabilitation," in 2017 IEEE 30th International Symposium on Computer-Based Medical Systems (CBMS). IEEE, jun 2017. [Online]. Available: https://doi.org/10.1109/ cbms.2017.89

[4] R. V. Aranha, "EasyAffecta: um framework baseado em computação afetiva para adaptação automática de jogos sérios para reabilitação motora," Master's thesis. [Online]. Available: https://doi.org/10.11606/d.100.2017.tde-24072017-083504

[5] R. Tadayon, A. Amresh, T. McDaniel, and S. Panchanathan, "Real-time stealth intervention for motor learning using player flow-state," in 2018 IEEE 6th International Conference on Serious Games and Applications for Health (SeGAH), 2018, pp. 1-8.

[6] A. Nagle, R. Riener, and P. Wolf, "Personality-based reward contingency selection: A player-centered approach to gameplay customization in a serious game for cognitive training," Entertainment Computing, vol. 28, pp. 70-77, dec 2018. [Online]. Available: https://doi.org/10.1016/j.entcom.2017.10. 005

[7] T. Alves, C. Martinho, and R. Prada, "Towards incorporating personality in serious games for health," in 2019 11th International Conference on Virtual Worlds and Games for Serious Applications (VS-Games), 2019, pp. 1-4.

[8] J. Brich, "Motivational game design factors in player-game adaptivity," in Extended Abstracts Publication of the Annual Symposium on Computer-Human Interaction in Play, ser. CHI PLAY ' 17 Extended Abstracts. New York, NY, USA: Association for Computing Machinery, 2017, p. 683-686. [Online]. Available: https://doi.org/10.1145/3130859.3133222

[9] R. V. Aranha, R. Nakamura, R. Tori, and F. L. S. Nunes, "Personality traits impacts in virtual reality's user experience," in 2018 20th Symposium on Virtual and Augmented Reality (SVR). IEEE, oct 2018. [Online]. Available: https://doi.org/10.1109/svr.2018.00019

[10] R. V. Aranha, C. G. Correa, and F. L. S. Nunes, "Adapting software with affective computing: a systematic review," IEEE Transactions on Affective Computing, pp. 1-1, 2019. [Online]. Available: https://doi.org/10.1109/taffc.2019.2902379 